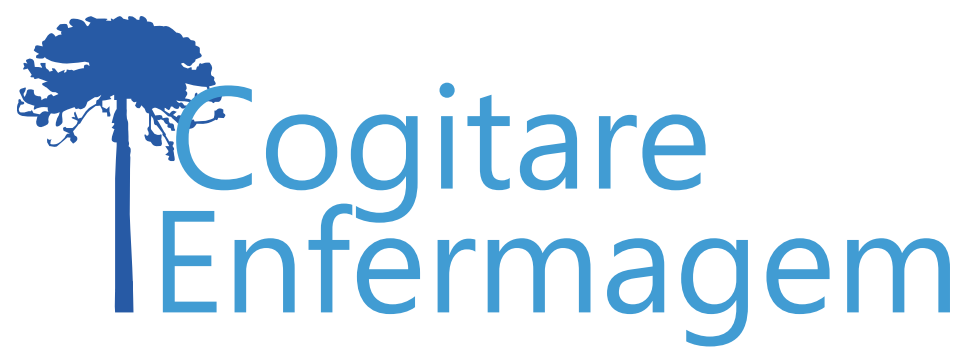

\title{
SÍNDROME DE BURNOUT EM PRECEPTORES E RESIDENTES VINCULADOS A PROGRAMAS DE RESIDÊNCIA EM SAÚDE DA FAMÍLIA
}

Luana Silveira ${ }^{1}$, Cristine Neves Bez², Erádio Gonçalves Júnior ${ }^{3}$, Caroline Cechinel Peiter ${ }^{4}$, Greici Capellari Fabrizzio ${ }^{5}$, José Luís Guedes dos Santos ${ }^{6}$

\section{RESUMO}

Objetivo: identificar a presença de Burnout e seus fatores associados em preceptores e residentes de Programas de Residência.

Método: estudo transversal realizado com 64 preceptores e residentes no contexto da Atenção Primária à Saúde, de outubro a novembro de 2016. Utilizou-se formulário socioprofissional e o questionário Maslach Burnout Inventory. Para análise dos dados, aplicaram-se os testes Exato de Fisher e Kruskal-Wallis. Considerou-se o nível de significância de $5 \%(p<0,05)$.

Resultados: os participantes apresentaram sentimento de Incompetência Profissional, Desgaste Emocional e Despersonalização moderados, com médias de 23,1; 17,5 e 4,1, respectivamente. Evidenciou-se associação entre as dimensões do Maslach Burnout Inventory e a variável Distrito Sanitário.

Conclusão: não foi identificada a presença de Burnout entre os participantes. No entanto, níveis moderados de sentimento de incompetência profissional, desgaste emocional e despersonalização podem influenciar negativamente a qualidade da assistência prestada. Esta pesquisa contribui para o conhecimento pré-existente por envolver residentes e preceptores multiprofissionais.

DESCRITORES: Enfermagem de Atenção Primária; Atenção Primária à Saúde; Esgotamento Profissional; Internato e Residência; Síndrome de Burnout.

\section{COMO REFERENCIAR ESTE ARTIGO:}

Silveira L, Bez CN, Gonçalves Júnior E, Peiter CC, Fabrizzio GC, Santos JLG dos. Síndrome de burnout em preceptores e residentes vinculados a programas de residência em saúde da família. Cogitare enferm. [Internet]. 2020 [acesso em "colocar data de acesso, dia, mês abreviado e ano"]; 25. Disponível em: http://dx.doi. org/10.5380/ce.v25i0.67248.

Este obra está licenciado com uma Licença Creative Commons Atribuição 4.0 Internacional.

${ }^{1}$ Enfermeira. Mestranda em Enfermagem. Universidade Federal de Santa Catarina. Florianópolis, SC, Brasil. () ${ }^{2}$ Odontóloga. Especialista em Saúde da Família. Universidade Federal de Santa Catarina. Florianópolis, SC, Brasil. 9 ${ }^{3}$ Fisioterapeuta. Mestre em Fisioterapia. Universidade do Estado de Santa Catarina. Florianópolis, SC, Brasil. (0) ${ }^{4}$ Enfermeira. Doutoranda em Enfermagem. Universidade Federal de Santa Catarina. Florianópolis, SC, Brasil. $\bigcirc$ ${ }^{5}$ Enfermeira. Doutoranda em Enfermagem. Universidade Federal de Santa Catarina. Florianópolis, SC, Brasil. $(0$ ${ }^{6}$ Enfermeiro. Doutor em Enfermagem. Docente de Enfermagem da Universidade Federal de Santa Catarina. Florianópolis, SC, Brasil. 


\title{
BURNOUT SYNDROME IN PRECEPTORS AND RESIDENTS LINKED TO FAMILY HEALTH RESIDENCY PROGRAMS
}

\begin{abstract}
Objective: to identify the Burnout and its associated factors in preceptors and residents of Residency Programs.

Method: a cross-sectional study carried out with 64 preceptors and residents in the context of Primary Health Care, from October to November 2016. A socio-professional form and the Maslach Burnout Inventory questionnaire were used. For data analysis, Fisher's Exact and Kruskal-Wallis tests were applied. The significance level was set at $5 \%(p<0.05)$.

Results: the participants showed moderate feelings of Professional Incompetence, Emotional Exhaustion and Depersonalization, with averages of 23.1; 17.5 and 4.1, respectively. There was an association between the dimensions of the Maslach Burnout Inventory and the Health District variable.

Conclusion: Burnout among the participants was not found. However, moderate levels of feeling of professional incompetence, emotional distress and depersonalization can negatively influence the quality of care provided. This research contributes to the pre-existing knowledge by involving residents and multi-professional tutors.
\end{abstract}

DESCRIPTORS: Primary Care Nursing; Primary Health Care; Burnout, Professional; Internship and Residency; Burnout, Psychological.

\section{SÍNDROME DE BURNOUT EN PRECEPTORES Y RESIDENTES ASOCIADOS A PROGRAMAS DE RESIDENCIA EN SALUD DE LA FAMILIA}

\section{RESUMEN:}

Objetivo: identificar la presencia de Burnout y los factores asociados en preceptores y residentes de Programas de Residencia.

Método: estudio transversal que se realizó con 64 preceptores y residentes en el contexto da Atención Básica a la Salud, de octubre a noviembre de 2016. Se utilizó formulario socio profesional y cuestionario Maslach Burnout Inventory. Para análisis de los datos, se aplicaron las pruebas Exacta de Fisher y Kruskal-Wallis. Se consideró el nivel de significancia de 5\% $(p<0,05)$.

Resultados: los participantes presentaron sentimiento de Incompetencia Profesional, Agotamiento Emocional y Despersonalización moderados, con promedios de 23,1; 17,5 y 4,1, respectivamente. Se constató asociación entre las dimensiones del Maslach Burnout Inventory y la variable Distrito Sanitario.

Conclusión: no se identificó la presencia de Burnout entre los participantes. Sin embargo, niveles moderados de sentimiento de incompetencia profesional, agotamiento emocional y despersonalización pueden influenciar negativamente la cualidad de la asistencia prestada. Esta investigación contribuye para el conocimiento preexistente por involucrar residentes y preceptores multi profesionales.

DESCRIPTORES: Enfermería de Atención Básica; Atención Básica a la Salud; Agotamiento Profesional; Internado y Residencia; Síndrome de Burnout. 
O aumento do escopo de atuação e responsabilização dos profissionais da Atenção Primária à Saúde (APS) no Brasil foi fortalecido a partir dos anos 2000, como resultado da expansão da Estratégia Saúde da Família (ESF). O modelo de atenção, até então hospitalocêntrico e baseado na doença, passou da centralidade no profissional médico à integração dos diferentes saberes multiprofissionais, em coerência com o modelo de redes de atenção à saúde (RAS), que considera a APS como ordenadora da rede. Nesse contexto, a responsabilização dos profissionais da APS aumentou gradativamente, envolvendo atribuições referentes à oportunização de acesso, longitudinalidade e continuidade da assistência integral ao indivíduo e à população(1,2).

Frente à necessidade de ampliação da atuação e qualificação de práticas avançadas dos profissionais da APS, a Residência em Saúde têm sido considerada uma modalidade ideal para formação de profissionais para o Sistema Único de Saúde (SUS) ${ }^{(3,4)}$. Os profissionais, em geral recém graduados, buscam a residência em saúde para adquirir experiência, excelência profissional e título de especialista na área escolhida. O objetivo dos Programas de Residência em Medicina de Família e Comunidade (PRM/MFC) e Multiprofissional em Saúde da Família (PREMULTISF) é formar profissionais por meio da educação em serviço, sob a lógica aprender-fazendo, para atuar na APS e demais pontos de atenção da RÁS, buscando qualificá-los para o desempenho de suas atividades no SUS e visando ao alcance das competências técnicas, políticas e éticas da $E S F^{(4)}$.

No Brasil, os programas de residência preveem uma carga horária mínima de 5.760 horas, distribuídas em 60 horas semanais, das quais $20 \%$ são destinadas às atividades teóricas e $80 \%$ às atividades práticas e teórico-práticas. $O$ desenvolvimento das habilidades e competências previstas para o residente durante o curso ocorre de maneira supervisionada por preceptores, tutores, supervisores pedagógicos e de estágio e outros sujeitos corresponsáveis pelos programas. Os preceptores são profissionais da saúde inseridos nos mesmos locais de trabalho dos residentes, neste caso, as Unidades Básicas de Saúde (UBS) $)^{(4)}$. Nesse sentido, as responsabilidades de estudante esperadas para o residente e as atribuiç̃ões de supervisão para o profissional preceptor se somam à carga de trabalho e às funções assistenciais inerentes à atuação na APS.

A atuação profissional na área da saúde ocorre de modo intenso, em que, além da alta carga de trabalho e da frequente acumulação de vínculo empregatício, há também intenso fardo emocional derivado do acompanhamento do processo saúde e doença. Frente a isso, o potencial de desencadeamento de estresse ocupacional resultante da dinâmica de trabalho dos profissionais da área da saúde nos diferentes contextos de atuação vem sendo amplamente discutido na literatura ${ }^{(5,6)}$. Nesse contexto, o estresse acontece como resultado de uma situação que excede as forças de adaptação e resiliência do indivíduo ${ }^{(7)}$.

A insuficiência de estratégias para superar os fatores estressantes que envolvem a atividade laboral pode ter como resultados diversas alterações psicossomáticas, dentre elas a Síndrome de Burnout (SB) ${ }^{(7)}$. A definição mais consolidada da SB a considera como uma reação à tensão emocional crônica motivada a partir do contato direto com outros seres humanos quando estes estão preocupados ou com problemas ${ }^{(8)}$, tendo como principais características o desgaste emocional, a despersonalização do cuidado prestado e o sentimento de incompetência profissional(9). Na Itália, a estrutura, validade e confiabilidade da escala foi testada. A análise forneceu suporte para uma versão de 20 itens e manteve as três categorias originais( ${ }^{(9)}$.

No Brasil e em outros países, a SB vem sendo estudada no contexto dos profissionais de saúde, explorando a prevalência da síndrome entre profissionais da APS ${ }^{(8,10-12)}$, contexto hospitalar ${ }^{(5-7,9,13-15)}$, e até mesmo entre residentes em saúde ${ }^{(16,17)}$. No entanto, a discussão sobre a SB entre membros de programas de residência, incluindo preceptores e residentes no âmbito da APS, ainda representa uma lacuna a ser explorada. Assim, o objetivo deste estudo foi identificar a presença de Burnout e seus fatores associados em preceptores e 
residentes de Programas de Residência.

\section{MÉTODO}

Trata-se de um estudo transversal, com coleta de dados realizada de outubro a novembro de 2016 em um PREMULTISF e um PRM/MFC vinculados a uma instituição pública de ensino de pós-graduação no município de Florianópolis-SC, tendo como participantes os residentes e preceptores de um PREMULTISF e um PRM/MFC.

A amostragem foi do tipo não probabilística, em que foram convidados a participar do estudo todos os 41 residentes e 51 preceptores integrantes dos programas. Como critérios de inclusão, foram considerados residentes ingressantes da turma 2015-2017, e preceptores vinculados aos dois programas. Foram excluídos do estudo aqueles que não responderam aos instrumentos encaminhados por e-mail.

Os pesquisadores abordaram inicialmente os participantes em reuniões periódicas pré-estabelecidas pelos programas. Nesta abordagem, procurou-se elucidar a temática e os objetivos da pesquisa, realizando o convite de participação no estudo e a coleta de endereços de e-mail dos interessados. Em um segundo momento, foram encaminhados via plataforma Google $®$ os seguintes documentos: Termo de Consentimento Livre e Esclarecido (TCLE), formulário socioprofissional e o questionário de Maslach Burnout Inventory (MBI), versão Human Service Survey (HSS)(18). Cabe ressaltar que todos os instrumentos foram autoaplicáveis. Do total de 92 residentes e preceptores vinculados aos programas, 28 não responderam ao questionário. Desse modo, a amostra da pesquisa foi composta por 64 participantes, sendo 34 residentes e 30 preceptores das áreas profissionais de educação física, enfermagem, farmácia, fisioterapia, medicina, nutrição, odontologia e serviço social.

Oformulário socioprofissional abordou as variáveis: idade, sexo, categoria profissional, função (preceptor ou residente), tempo de atuação profissional e Distrito Sanitário (DS) à qual pertence a UBS em que o profissional estava inserido. $O$ instrumento escolhido para avaliação da SB, o MBI-HSS, foi elaborado por Christina Maslach e Susan Jackson em 1981, sendo posteriormente adaptado e validado para a realidade brasileira ${ }^{(18,19)}$.

O MBI-HSS é um instrumento do tipo Likert, com uma escala de cinco pontos em que se assinala zero para nunca, um para algumas vezes ao ano, dois para algumas vezes ao mês, três para algumas vezes na semana, e quatro para diariamente. É composto por 22 itens distribuídos em três subescalas, independentes entre si, em que nove itens são referentes ao Desgaste Emocional (DE), cinco à Despersonalização (DP) e oito à Falta de Realização Profissional ou Sentimento de Incompetência Profissional (IP). As primeiras duas dimensões, DE e DP, tem escore positivo, ou seja, quanto maior a pontuação, maior o nível de $S B$, enquanto a dimensão IP se apresenta de maneira reversa, de modo que quanto menor o escore, maior o nível de $\mathrm{SB}^{(20)}$.

Os dados foram tabulados em planilha do Excel® e, para interpretar as pontuações alcançadas nas três escalas, foram assumidos os pontos de corte apresentados no Quadro $1^{(14)}$. 
Quadro 1 - Valores da escala do MBI-HSS. São Paulo, SP, Brasil, 2015

\begin{tabular}{|c|c|c|c|}
\hline \multicolumn{2}{|c|}{ Dimensões } & Pontos de corte \\
\hline Desgaste emocional & Despersonalização & $\begin{array}{c}\text { Sentimento de } \\
\text { Incompetência Profissional }\end{array}$ & \\
\hline$\leq 10$ & $\leq 2$ & $>27$ & Baixo \\
\hline 11 a 21 & 3 a 8 & 21 a 27 & Moderado \\
\hline$>21$ & $>8$ & $\leq 20$ & Alto nível \\
\hline
\end{tabular}

Fonte: Ferreira, Lucca $(2015)^{(14)}$.

Os dados foram processados no programa Statistical Package for the Social Sciences (SPSS) versão 23 e analisados por meio de estatística descritiva e inferencial. Para verificar associação entre as variáveis, foram aplicados o teste Exato de Fisher e o teste não paramétrico de Kruskall Wallis. Considerou-se o nível de $5 \%$ de significância $(p<0,05)$.

O projeto de pesquisa foi submetido à Comissão de Acompanhamento dos Projetos de Pesquisa em Saúde do município e ao Comitê de Ética e Pesquisa com Seres Humanos, sendo aprovada pelo parecer 1.686.785.

\section{RESULTADOS}

As características predominantes dos participantes foi idade menor que 30 anos, sexo feminino e tempo de atuação na profissão entre dois e cinco anos. As áreas de formação com maior número de participantes foram Enfermagem e Medicina. As características socioprofissionais dos participantes da pesquisa estão representadas na Tabela 1.

Tabela 1 - Características socioprofissionais de preceptores e residentes dos Programas de Residência Multiprofissional e Médica em Saúde da Família. Florianópolis, SC, Brasil, 2016 (continua)

\begin{tabular}{lll} 
Características socioprofissionais & $\mathbf{N}$ & $\%$ \\
\hline Idade (anos completos) & & \\
\hline$<30$ anos & 25 & 39,1 \\
\hline Entre 30 e 35 anos & 21 & 32,8 \\
\hline Entre 36 e 40 anos & 11 & 17,2 \\
\hline$>40$ anos & 7 & 10,9 \\
\hline Sexo & & \\
\hline Feminino & 48 & 75 \\
\hline Masculino & 16 & 25 \\
\hline Categoria profissional & & \\
\hline Enfermagem & 21 & 32,8 \\
\hline
\end{tabular}




\begin{tabular}{|c|c|c|}
\hline Medicina & 17 & 26,5 \\
\hline Odontologia & 10 & 15,6 \\
\hline Fisioterapia & 5 & 7,8 \\
\hline Educação Física & 4 & 6,3 \\
\hline Farmácia & 3 & 4,7 \\
\hline Nutrição & 3 & 4,7 \\
\hline Serviço Social & 1 & 1,6 \\
\hline \multicolumn{3}{|l|}{ Função } \\
\hline Residente & 34 & 53,1 \\
\hline Preceptor & 30 & 46,9 \\
\hline \multicolumn{3}{|l|}{ Distrito sanitário } \\
\hline Sul & 24 & 37,5 \\
\hline Continente & 15 & 23,4 \\
\hline Leste & 14 & 21,9 \\
\hline Centro & 6 & 9,4 \\
\hline Norte & 5 & 7,8 \\
\hline \multicolumn{3}{|c|}{ Tempo de atuação na profissão } \\
\hline 2 a 5 anos & 36 & 56,3 \\
\hline 6 a 10 anos & 15 & 23,4 \\
\hline$>10$ anos & 13 & 20,3 \\
\hline
\end{tabular}

Fonte: Os autores (2016).

Não foi identificada a presença de SB entre os preceptores e residentes. Os participantes apresentaram IP, DE e DP moderados, com média respectivamente de 23,1; 17,5 e 4,1. A análise descritiva das dimensões do MBI-HSS, referentes à IP, DE e DP, pode ser visualizada na Tabela 2.

Tabela 2 - Dimensões da Síndrome de Burnout em preceptores e residentes dos Programas de Residência Multiprofissional e Médica em Saúde da Família. Florianópolis, SC, Brasil, 2016 (continua)

\begin{tabular}{|c|c|c|c|c|c|c|}
\hline Dimensão & Nível das dimensões & $\mathrm{N}=64(\%)$ & $\begin{array}{l}\text { Min- } \\
\text { Máx. }\end{array}$ & Média & $\begin{array}{l}\text { Desvio } \\
\text { padrão }\end{array}$ & Variância \\
\hline \multirow{3}{*}{$\begin{array}{l}\text { Sentimento de } \\
\text { incompetência } \\
\text { profissional }\end{array}$} & Baixo & $19(29,7)$ & \multirow{3}{*}{$9-32$} & \multirow{3}{*}{23,1} & \multirow{3}{*}{5,9} & \multirow{3}{*}{34,3} \\
\hline & Moderado & $29(45,3)$ & & & & \\
\hline & Alto & $16(25)$ & & & & \\
\hline \multirow{3}{*}{ Desgaste emocional } & Baixo & $14(21,9)$ & \multirow{3}{*}{$4-34$} & \multirow{3}{*}{17,5} & \multirow{3}{*}{7,6} & \multirow{3}{*}{58,3} \\
\hline & Moderado & $33(51,5)$ & & & & \\
\hline & Alto & $17(26,6)$ & & & & \\
\hline
\end{tabular}




\begin{tabular}{lllllll}
\hline \multirow{2}{*}{ Despersonalização } & \multicolumn{2}{l}{ Baixo } & $27(42,2)$ & & & \\
\cline { 2 - 3 } & Moderado & $28(43,7)$ & $9(14,1)$ & 4,1 & 3,5 & 12,3
\end{tabular}

Fonte: Os autores (2016).

Quanto às associações entre as dimensões do MBI-HSS, evidenciou-se associação positiva e estatisticamente significante entre o IP e DE entre IP e DP, e entre DE e DP (Tabela 3).

Tabela 3 - Análise das associações entre as dimensões da Síndrome de Burnout em preceptores e residentes dos Programas de Residência Multiprofissional e Médica em Saúde da Família. Florianópolis, SC, Brasil, 2016

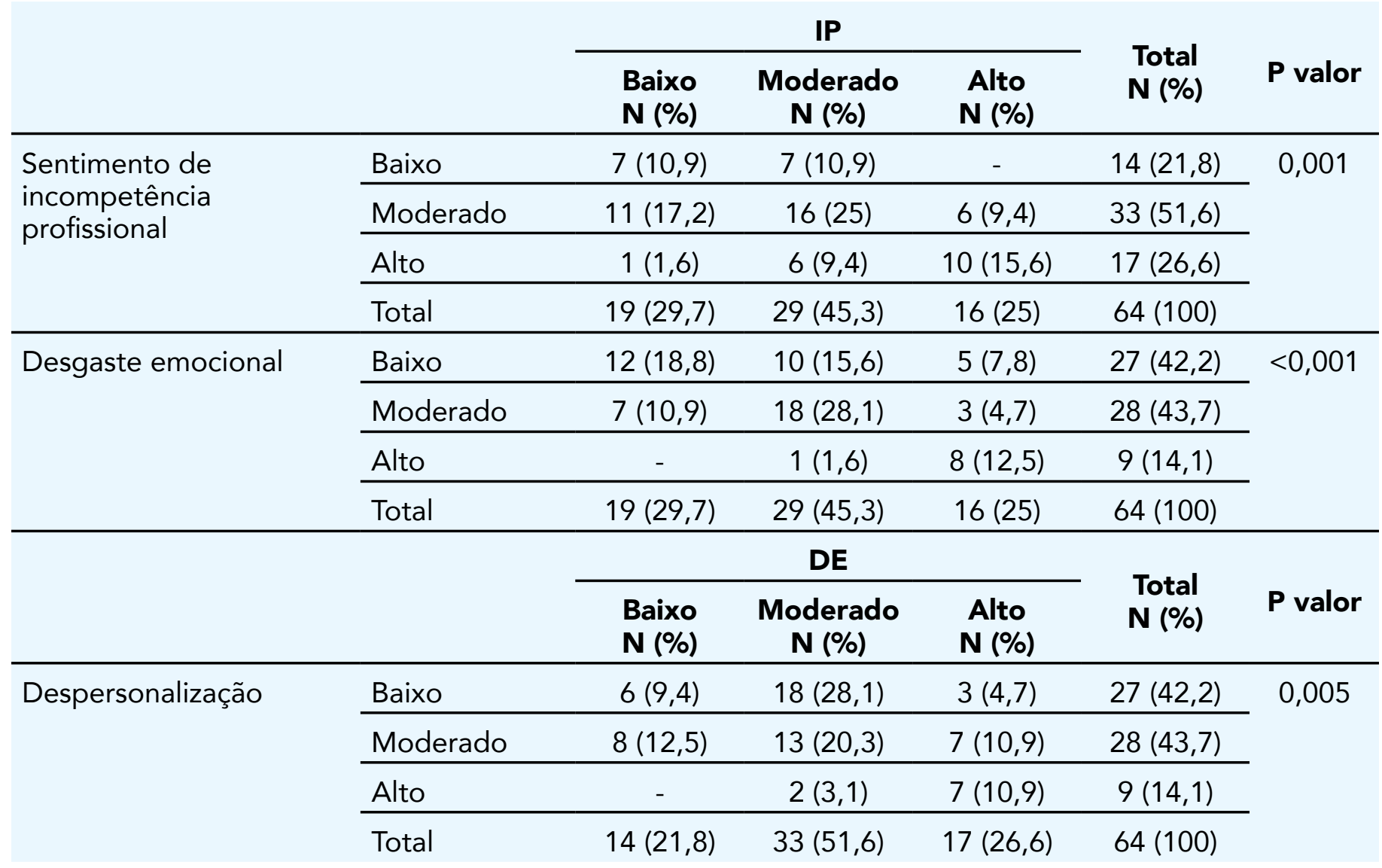

Fonte: Os autores (2016).

Quanto às associações entre as variáveis sociodemográficas e profissionais e as dimensões da Síndrome de Burnout, não foi observada associação entre os níveis de $I P$, DE e DP frente às variáveis idade, sexo, categoria profissional, função e tempo de atuação profissional. Em relação ao distrito sanitário da UBS, foi observada dif̧erença estatisticamente significante $(p=0,031)$ entre o nível de IP e os diferentes distritos sanitários do município. $\mathrm{O}$ distrito sanitário Sul teve destaque com maior nível de IP, como representa a Tabela 4. 
Tabela 4 - Associação entre sentimento de Incompetência Profissional e Distrito Sanitário. Florianópolis, SC, Brasil, 2016

\begin{tabular}{lcccc} 
Distrito Sanitário & \multicolumn{2}{c}{ Sentimento de incompetência profissional } & Total \\
\cline { 2 - 4 } & $\begin{array}{c}\text { Baixo } \\
\mathbf{N}(\%)\end{array}$ & $\begin{array}{c}\text { Moderado } \\
\mathbf{N}(\%)\end{array}$ & $\begin{array}{c}\text { Alto } \\
\mathbf{N}(\%)\end{array}$ & \begin{tabular}{c} 
N (\%) \\
\hline Centro
\end{tabular} \\
\hline Continente & $2(33,3)$ & $2(33,3)$ & $2(33,3)$ & $6(100)$ \\
\hline Leste & $3(20)$ & $10(66,7)$ & $2(13,3)$ & $15(100)$ \\
\hline Norte & $4(28,6)$ & $9(64,3)$ & $1(7,1)$ & $14(100)$ \\
\hline Sul & $1(20)$ & $3(60)$ & $1(20)$ & $5(100)$ \\
\hline Total & $6(25)$ & $5(20,8)$ & $13(54,2)$ & $24(100)$ \\
\hline
\end{tabular}

Fonte: Os autores (2016).

\section{DISCUSSÃO}

Dentre os participantes do estudo, foi observada maior prevalência de membros menores que 35 anos. Em estudo que buscou identificar o perfil sociodemográfico e acadêmico de 37 residentes multiprofissionais em saúde, observou-se que 51,4\% dos participantes encontravam-se na faixa etária entre 25 e 29 anos, corroborando com achados deste estudo. Isso pode estar relacionado ao fato de que os profissionais recém formados identificam na residência uma oportunidade de adquirir experiência e habilidade técnica na área de interesse, o que resulta no frequente ingresso nos cursos de residência logo após a graduação(21).

A maior presença de profissionais das áreas de Enfermagem e Medicina devese à magnitude da força de trabalho desses profissionais nos serviços de saúde e, consequentemente, ao maior número de vagas previstas em edital para esses profissionais nos programas pesquisados. Portanto, profissionais dessas áreas representam o maior número de residentes do programa e, em decorrência disso, têm o maior número de preceptores da mesma categoria profissional.

O contexto estudado apresenta o terceiro maior Índice de Desenvolvimento Humano Municipal do país, sendo o primeiro dentre as capitais ${ }^{(22)}$. Além disso, o município representa um modelo de referência nacional em boas práticas no contexto da APS(23), o que pode ter contribuído para a ausência da SB entre os participantes.

Ainda assim, os participantes apresentaram índices de IP, DE e DP moderados, indicando a necessidade de reflexão sobre as condições de formação desses profissionais. Infere-se, desse modo, que esses valores poderiam ser influenciados negativamente de acordo com o ambiente de trabalho da realidade investigada, tendo em vista a heterogeneidade dos municípios brasileiros e sua responsabilidade sobre o nível primário da atenção. Nesse sentido, é importante ressaltar a necessidade de outros estudos, para avaliar a realidade vivida pelos membros de programas de residência nos diferentes cenários da APS.

Apesar de não ter sido evidenciado SB em residentes e preceptores, estudos têm demonstrado sua prevalência em profissionais que trabalham no contexto da APS(11-15). Considerando a organização do SUS segundo a lógica de RAS, sendo estas ordenadas pela APS, as condições de saúde dos profissionais deste nível de atenção repercutem na qualidade do cuidado ofertado em toda a rede ${ }^{(8)}$. As mudanças recentes de abordagem 
na ESF requerem preparo e capacitação para enfrentá-las de modo satisfatório. Junto a esta exigência, as UBS, muitas vezes, estão inseridas em territórios perigosos e insalubres, marcados por desigualdades sociais e pobreza, o que pode favorecer o surgimento de sofrimento psíquico dos profissionais envolvidos ${ }^{(24)}$.

Em alguns casos, além da carga horária de 60 horas semanais, os residentes estão expostos a fatores como o desrespeito às necessidades básicas do processo ensinoaprendizagem, ausência de acompanhamento do preceptor, ausência de momentos para estudo e descanso ${ }^{(16,17)}$. Os residentes expostos aos estressores da formação, somados aos da profissão, podem desenvolver a SB quando não utilizam estratégias adequadas para minimizar ou eliminar o estressor. Nesse sentido, danos pessoais, familiares, institucionais e sociais são possíveis nessa população ${ }^{(25)}$. Ao encontro disso, a exigência de um conjunto de funções e tarefas realizadas pelos preceptores, em um contexto com influências da situação social, econômica e política, vivido no trabalho na ESF, contribui para a propensão a diferentes níveis de estresse que podem levar à $\mathrm{SB}^{(26)}$.

$\mathrm{Na}$ análise da presença de SB entre os participantes do estudo, foi identificada associação entre as três dimensões do MBI. O sentimento de IP pode ser resultado de uma percepção de fracasso frente às possibilidades terapêuticas dos usuários assistidos, fazendo com que o profissional se torne incapaz de diferenciar as fragilidades do sistema de saúde de seus próprios limites de autonomia e governabilidade ${ }^{(5)}$. Está associado à baixa autoestima, sentimento de inadequação, insuficiência e desmotivação, o que reflete em diminuição do engajamento e comprometimento com a qualidade do trabalho realizado, e influencia negativamente a assistência prestada(27). Nesse sentido, podemos inferir que o sentimento de IP identificado nos preceptores e residentes é produto do desgaste emocional derivado das condições de trabalho, o que pode comprometer uma assistência humanizada e individualizada, resultando na despersonalização do cuidado.

Sendo assim, a prevenção e o tratamento da SB transcendem os benefícios individuais dos profissionais, refletindo no ambiente de trabalho e em melhores rendimentos das organizações. Como exemplo disso, destaca-se a melhor oferta de serviço, menos dias de afastamento do trabalho e melhor rendimento econômico(28).

A relação entre o sentimento de IP com desgaste e despersonalização do cuidado prestado por residentes e preceptores multiprofissionais em saúde da família, atrelada ao local de trabalho, sugere a interferência de diferentes processos de gestão como um fator contributivo para o desencadeamento da SB. Estudo realizado em cinco regiões do Brasil apontou que a insatisfação no trabalho neste cenário está vinculada a fatores como estrutura física inadequada, falta de recursos materiais, falhas na gestão, jornada de trabalho excessiva, falta de valorização do trabalho e déficit salarial(29).

A SB tem sido entendida não como um problema do indivíduo, mas do ambiente social no qual ele desempenha suas habilidades laborais, tendo destaque a influência da gestão organizacional no trabalho(17). A falta de comprometimento e planejamento dos gestores na continuidade das ações em saúde, atrelada à influência política nos serviços, dificulta a implementação da Política Nacional de Atenção Básica (PNAB). Isso reflete no cotidiano assistencial da ESF e dificulta sua resolutividade. Existe, ainda, a alta rotatividade de gestores comprometidos e capacitados por questões meramente políticas, dificultando a continuidade de boas práticas e a prestação de serviços de melhor qualidade ${ }^{(24)}$.

O sentimento de IP foi identificado como moderado em três distritos sanitários e alto em um deles. Os ambientes organizacionais são moldados por fatores sociais, políticos e econômicos, e estão em constante transformação. Quando estas transformações resultam em altas demandas com poucos recursos, podem influenciar o esgotamento profissional. Além disso, as relações terapêuticas, que requerem atenção contínua e intensa de contato emocional e pessoal, no caso da APS que tem como objetivo a longitudinalidade do cuidado, podem ao mesmo tempo ser recompensadoras e estressoras ${ }^{(27)}$.

Não houve associação frente à idade, sexo, categoria profissional e função com o sentimento de DE, DP e IP. Estudo realizado com trabalhadores das ESF em um 
município no Sul do Brasil aponta que transtornos mentais comuns foram mais frequentes nos profissionais entre 36 a 45 anos e enfermeiros, sem, entretanto, mostrar diferenças estatísticas significativas em comparação com as demais faixas de idade avaliadas e categorias profissionais. Com relação ao sexo e $\mathrm{SB}$, há divergências na literatura, sendo que alguns autores observaram maior risco de burnout em mulheres ${ }^{(30)}$ e outros em homens, e ainda aqueles que não encontraram associação à variável sexo ${ }^{(8,12)}$.

Em relação ao tempo de atuação na profissão, estudo que analisou a presença de SB em profissionais da APS evidenciou que, quanto maior o tempo de atuação na profissão, menor a propensão à SB, uma vez que este fator favorece o profissional por proporcionar mais experiência e familiaridade com o contexto. Em contrapartida, quanto menor o tempo de atuação, maiores as inseguranças frente à tomada de decisão e a dificuldade em lidar com os fatores estressantes, indicando que o menor de tempo de atuação na profissão tem associação com a $\mathrm{SB}^{(10)}$.

Como limitações da pesquisa, pontua-se a adoção de uma amostra do tipo não probabilística a partir de inclusão de residentes e preceptores de dois programas de residência de um mesmo município, o que compromete a generalização dos resultados. Apesar disso, os achados do estudo fornecem subsídios importantes para a discussão da saúde do trabalhador na APS.

\section{CONSIDERAÇÕES FINAIS}

Não foi identificada presença de Burnout em preceptores e residentes multiprofissionais, no entanto os participantes apresentaram sentimento de IP, DE e DP moderados.

Este estudo contribui para os programas de residência multiprofissional em saúde da família e medicina de família e comunidade, bem como para o cenário da atenção primária. A pesquisa avança no conhecimento pré-existente por envolver preceptores e residentes multiprofissionais e apontar fatores relacionados ao sentimento de IP que podem influenciar diretamente no cuidado prestado neste contexto.

\section{REFERÊNCIAS}

1. Fertonani HP, Pires DEP de, Biff D, Sherer MD dos A. Modelo assistencial em saúde: conceitos e desafios para a atenção básica brasileira. Ciênc. saude colet. [Internet]. 2015 [acesso em 21 nov 2016]; 20(6). Disponível em: http://dx.doi.org/10.1590/1413-81232015206.13272014.

2. Backes DS, Backes MS, Erdmann AL, Büscher A. O papel profissional do enfermeiro no Sistema Único de Saúde: da saúde comunitária à estratégia de saúde da família. Ciênc. saude colet. [Internet]. 2012 [acesso em 9 maio 2019]; 17(1). Disponível em: http://dx.doi.org/10.1590/S1413-81232012000100024.

3. Organização Pan-Americana da Saúde (OPAS). Ampliação do papel dos enfermeiros na atenção primária à saúde [Internet]. Washington: OPAS; 2018 [acesso 23 maio 2018]. Disponível em: http://iris. paho.org/xmlui/handle/123456789/34960.

4. Martins GDM, Caregnato RCA, Barroso VLM, Ribas DCP. Implementação de residência multiprofissional em saúde de uma universidade federal: trajetória histórica. Rev. Gaúcha Enferm. [Internet]. 2016 [acesso em 10 maio 2019]; 37(3). Disponível em: http://www.scielo.br/scielo.php?script=sci arttext\&pid=S1983-14472016000300405.

5. Fernandes LS, Nitsche MJT, Godoy I de. Associação entre Síndrome de burnout, uso prejudicial de álcool e tabagismo na Enfermagem nas UTIs de um hospital universitário. Ciênc. saude coletit. 
[Internet]. 2018 [acesso em 10 maio 2019]; 23(1). Disponível em: http://dx.doi.org/10.1590/141381232018231.05612015.

6. Prudente JAB, Salum NC, Gelbcke FL, Lorenzetti J, Schier J. O desgaste de trabalhadores de enfermagem no cuidado a pacientes onco-hematológicos hospitalizados. Cogitare enferm. [Internet]. 2015 [acesso em 09 maio 2019]; 20(1). Disponível em: http://dx.doi.org/10.5380/ce.v20i1.38299.

7. Andolhe R, Barbosa RL, Oliveira EM de, Costa ALS, Padilha KG. Stress, coping and burnout among Intensive Care Unit nursing staff: associated factors. Rev. Esc. Enferm. USP [Internet]. 2015 [acesso em 11 maio 2019]; 49(n.esp). Disponível em: https://doi.org/10.1590/S0080-623420150000700009.

8. Morelli SGS, Sapede M, Silva ATC da. Burnout em médicos da Atenção Primária: uma revisão sistemática. Rev. bras. med. fam. comunidade. [Internet]. 2015 [acesso em 11 maio 2019]; 10(34). Disponível em: https://doi.org/10.5712/rbmfc10(34)958.

9. Loera B, Converso D, Viotti S. Evaluating the Psychometric Properties of the Maslach Burnout Inventory-Human Services Survey (MBI-HSS) among Italian Nurses: how many factors must a researcher consider? PLoS ONE [Internet]. 2014 [acesso em 13 dez 2019]; 9(12). Disponível em: https://www.ncbi. nlm.nih.gov/pmc/articles/PMC4264862/pdf/pone.0114987.pdf.

10. Lima A de S, Farah BF, Bustamante-Teixeira MT. Análise da prevalência da síndrome de burnout em profissionais da atenção primária em saúde. Trab. educ. saúde [Internet]. 2018 [acesso em 10 maio 2019];16(1). Disponível em: http://dx.doi.org/10.1590/1981-7746-sol00099.

11. Navarro-González D, Ayechu-Díaz A, Huarte-Labiano I. Prevalencia del síndrome del burnout y factores asociados a dicho síndrome en los profesionales sanitários de atención primaria. Semergen: medicina de familia. [Internet]. 2015 [acesso em 11 maio 2019]; 41(4). Disponível em: https://doi. org/10.1016/j.semerg.2014.03.008.

12. Amiri M, Khosravi A, Eghtesadi AR, Sadeghi Z, Abedi G, Ranjbar M, et al. Burnout and its influencing factors among primary health care providers in the north east of Iran. PLoS ONE [Internet]. 2016 [acesso em 13 dez 2019]; 11(12). Disponível em: https://journals.plos.org/plosone/article?id=10.1371/journal. pone.0167648.

13. Cruz SP de la, Abellán MV. Professional burnout, stress and job satisfaction of nursing staff at a university hospital. Rev. Latino-Am. Enfermagem [Internet]. 2015 [acesso em 09 maio 2019]; 23(3). Disponível em: https://doi.org/10.1590/0104-1169.0284.2586.

14. Ferreira N do N, Lucca SR de. Síndrome de burnout em técnicos de enfermagem de um hospital público do Estado de São Paulo. Rev. bras. epidemiol. [Internet]. 2015 [acesso em 09 maio 2019]; 18(1). Disponível em: https://doi.org/10.1590/1980-5497201500010006.

15. Lima RA dos S, Souza Al de, Galindo RH, Feliciano KV de O. Vulnerabilidade ao burnout entre médicos de hospital público do Recife. Cienc. saude colet. [Internet]. 2013 [acesso em 10 maio 2019]; 18(4). Disponível em: http://dx.doi.org/10.1590/S1413-81232013000400018.

16. Sponholz TCH, Sartori MT, Marques APS, Albuquerque GSC de, Silva MJ de S e, Perna P de O. Processo de trabalho na residência médica: a subordinação do ensino-aprendizagem à exploração da força de trabalho dos residentes. Trab. educ. saúde. [Internet]. 2016 [acesso em 10 maio 2019]; 14(1). Disponível em: http://dx.doi.org/10.1590/1981-7746-sol00025.

17. Guido L de A, Silva RM da, Goulart CT, Bolzan ME de O, Lopes LFD. Burnout Syndrome in multiprofessional residents of a public university. Rev. Esc. Enferm. USP [Internet]. 2012 [acesso em 11 maio 2019]; 46(6). Disponível em: http://dx.doi.org/10.1590/S0080-62342012000600027.

18. Lautert L. O desgaste profissional: estudo empírico com enfermeiras que trabalham em hospitais. Rev Gaúcha Enferm. [Internet]. 1997 [acesso em 11 maio 2019]; 18(12). Disponível em: https://seer.ufrgs.br/ RevistaGauchadeEnfermagem/article/view/4140.

19. Maslach C, Jackson SE. The measurement of experienced burnout. J Organ Behav. [Internet]. 1981 [acesso em 10 maio 2019]; (2)2. Disponível em: https://doi.org/10.1002/job.4030020205. 
20. Trindade L de L, Lautert L. Syndrome of Burnout among the workers of the Strategy of Health of the Family. Rev. Esc. Enferm. USP [Internet]. 2010 [acesso em 11 maio 2019]; 44(2). Disponível em: http:// dx.doi.org/10.1590/S0080-62342010000200005.

21. Goulart CT, Silva RM da, Bolzan MEO, Guido LA. Perfil sociodemográfico e acadêmico dos residentes multiprofissionais de uma universidade pública. Rev. RENE. [Internet]. 2012 [acesso em 11 maio 2019]; 13(1). Disponível em: http://www.periodicos.ufc.br/rene/article/view/3791/2994.

22. Instituto Brasileiro de Geografia e Estatística (IBGE). Florianópolis. [Internet] Florianópolis; IBGE; 2017 [acesso em 15 ago 2018]. Disponível em: https://cidades.ibge.gov.br/brasil/sc/florianopolis/panorama.

23. Almeida PF de, Gérvas J, Freire J-M, Giovanella L. Estratégias de integração entre atenção primária à saúde e atenção especializada: paralelos entre Brasil e Espanha. Saude debate. [Internet]. 2013 [acesso em 11 maio 2019]; 37(98). Disponível em: http://dx.doi.org/10.1590/S0103-11042013000300004.

24. Seidl H, Vieira SP, Fausto MCR, Lima RCD, Gagno J. Gestão do trabalho na atenção básica em saúde: uma análise a partir da perspectiva das equipes participantes do PMAQ-2012. Saude debate. [Internet]. 2014 [acesso 11 maio 2019]; 38(n.esp). Disponível em: http://dx.doi.org/10.5935/0103-1104.2014S008.

25. Silva $D$ de $P$ da, Silva $M$ de NRM de $O$. O trabalhador com estresse e intervenções para o cuidado em saúde. Trab. educ. saúde [Internet]. 2015 [acesso em 10 maio 2019]; 13(supl.1). Disponível em: http:// dx.doi.org/10.1590/1981-7746-sip00032.

26. Torres $\mathrm{P}$, Cobo J. Estrategias de gestión de la inteligencia emocional para la prevención del Sindrome de Burnout en docentes de aula. Educación en Contexto. [Internet]. 2016 [acesso em 10 maio 2019]; 2(n. esp). Disponível em: https://dialnet.unirioja.es/servlet/articulo?codigo=6296667.

27. Maslach C, Leiter MP. Understanding the burnout experience: recent research and its implications for psychiatry. World psychiatry. [Internet]. 2016 [acesso em 11 maio 2019]; 15(2). Disponível em: https://doi. org/10.1002/wps.20311.

28. Morales LS, Murillo LFH. Síndrome de Burnout. Med. leg. Costa Rica. [Internet]. 2015 [acesso em 11 maio 2019]; 32(1). Disponível em: https://www.scielo.sa.cr/pdf/mlcr/v32n1/art14v32n1.pdf.

29. Soratto J, Pires DEP de, Trindade LL, Oliveira JSA de, Forte ECN, Melo TP de. Insatisfação no trabalho de profissionais da saúde na estratégia saúde da família. Texto contexto-enferm. [Internet]. 2017 [acesso em 11 maio 2019]; 26(3). Disponível em: http://dx.doi.org/10.1590/0104-07072017002500016.

30. Moreira IJB, Horta JA, Duro LN, Borges DT, Cristofari AB, Chaves J, et al. Perfil sociodemográfico, ocupacional e avaliação das condições de saúde mental dos trabalhadores da Estratégia Saúde da Família em um município do Rio Grande do Sul, RS. Rev. bras. med. fam. comunidade. [Internet]. 2016 [acesso em 11 maio 2019]; 11(38). Disponível em: https://www.rbmfc.org.br/rbmfc/article/view/967.

Recebido: 03/06/2019

Finalizado: 14/04/2020

Editora associada: Susanne Elero Betiolli

\author{
Autor Correspondente: \\ Luana Silveira \\ Universidade Federal de Santa Catarina \\ Rd. Amaro Antônio Vieira, 2155 - 88034102 - Florianópolis, SC, Brasil \\ E-mail: luanasilveira2@gmail.com
}


Contribuição dos autores:

Contribuições substanciais para a concepção ou desenho do estudo; ou a aquisição, análise ou interpretação de dados do estudo - LS, CNB, EGJ, CCP, GCF

Elaboração e revisão crítica do conteúdo intelectual do estudo - LS, CNB, EGJ, CCP, GCF, JLGS

Aprovação da versão final do estudo a ser publicado - JLGS

Responsável por todos os aspectos do estudo, assegurando as questões de precisão ou integridade de qualquer parte do estudo - LS 